\title{
林线树种太白红杉种子萌发的生理生态特性
}

\begin{abstract}
张 玲
(北京大学环境学院生态学系、北京大学生态学研究与教育中心、北京大学地表过程分析与模拟教育部重点实验室, 北京 100871)

摘 要 太白红杉 (Larix chinensis) 是太白山的高山林线树种。通过在人工气候室内的试验, 研究了太白红杉种子 在 6 种不同的光照与温度组合处理条件下的萌发特性。结果表明: 在恒温和变温两种条件下, 交替光照对于种子 吸胀后的脱落酸 $(\mathrm{ABA})$ 和赤霉素 (GA) 有刺激作用。在恒温条件下, 持续光照对于种子吸胀后的生长素 (IAA)有刺 激作用, 而变温条件下交替光照对生长素有刺激作用。细胞分裂素( CTK) 的变化情况与 IAA 相反。光照条件相同 时, 恒温条件下的植物激素含量要高于变温条件下的含量, 说明恒温对于各种激素有刺激作用。在 $25{ }^{\circ} \mathrm{C}$ 环境下种 子的萌发率高于在 $12{ }^{\circ} \mathrm{C}$ 环境下的萌发率, 说明温度对于种子的萌发有重要作用。太白红杉种子的萌发受交替光 照 $\left(12 \mathrm{~h}\right.$ 光照 $/ 12 \mathrm{~h}$ 黑暗)的刺激 恒温 $\left(25^{\circ} \mathrm{C}\right)$ 条件下的种子萌发率高于变温 $\left(12{ }^{\circ} \mathrm{C} / 25{ }^{\circ} \mathrm{C}\right)$ 条件下的种子萌发率。 实验结果反映了内源激素在太白红杉种子萌发过程中起着重要作用。
\end{abstract}

关键词 太白红杉种子 光照 温度 萌发 激素 生理生态特性

\section{ECO-PHYSIOLOGICAL CHARACTERISTICS OF SEED GERMINATION OF LARIX CHINENSIS , A TIMBERLINE TREE}

\author{
ZHANG Ling \\ (Department of Ecology , and Key Laboratory for Earth Surface Processes of the Ministry of Education , Peking University , Beijing 100871 , China)
}

\begin{abstract}
Larix chinensis is a timberline tree species on Mt. Taibai in the Qinling mountains. To better understand climatic and physiological processes that control timberline formation , the eco-physiological characteristics of $L$. chinensis seeds were investigated in the laboratory by exposing seeds to different treatments of illumination and temperature. The major results are summarized below. An alternating photoperiod of $12 \mathrm{~h}$ light: $12 \mathrm{~h}$ dark increased phytohormone levels of $\mathrm{ABA}$ and $\mathrm{GA}$ in imbibed seeds during germination. Under conditions of constant temperatures of $25{ }^{\circ} \mathrm{C}$ or $12{ }^{\circ} \mathrm{C}$, constant illumination had a positive affect on phytohormone levels of IAA in imbibed seeds. Under alternating temperatures of $25{ }^{\circ} \mathrm{C}$ for $12 \mathrm{~h}$ and $12{ }^{\circ} \mathrm{C}$ for $12 \mathrm{~h}$, alternating illumination did not affect phytohormone contents of IAA. Changes in phytohormone levels of CTK showed an opposite response as IAA. Constant temperatures had a positive effect on phytohormone contents in imbibed seeds. Temperature was crucial for seed germination, and the rate of seed germination at a constant temperature of $25{ }^{\circ} \mathrm{C}$ was greater than at a constant temperature of $12{ }^{\circ} \mathrm{C}$. Alternating illumination $(12 \mathrm{~h}$ light $: 12 \mathrm{~h}$ dark) also had a strong, positive effect on seed germination. Otherwise the results showed that endogenous phytohormone levels were important for seed germination. The results indicated that temperature affected the germination rate and success of $L$. chinensis seeds, which is crucial for regeneration of $L$. chinensis , while illumination impacts were weak. Further studies are needed to better understand the underlying mechanisms of how temperature affects timberline formation on Mt. Taibai, Qinling mountains.
\end{abstract}

Key words Larix chinensis , Seed germination , Temperature , Illumination , Phytohormones

种子是种子植物的繁殖体，种子萌发特性对于 维持种群特定的结构与种群组分有着重要的作用。 植物群落的形成、演替与种子萌发特性有关，甚至可 以说在陆地植被的各种生态现象中都包含种子和幼 苗问题, 并通过有关种群过程 (如定居、扩散、遗传变 异)而影响到植被分布、动态和多样性 (Thompson， 1992)。因此, 研究植物种子的萌发能力和萌发过程 对于研究植被分布和植物种群动态十分重要。
太白红杉 (Larix chinensis) 属松科落叶松属的落 叶乔木, 秦岭特有种, 国家二级保护植物, 是秦岭太 白山的高山林线树种。太白红杉种子在较高海拔的 样地中, 也是土壤种子库中的优势成分之一 (张玲 等 ,2004)。太白山林线的形成机制是一个重要的研 究课题 林线树种的生理特性研究是其中的一个重 要方面(Tranquillini，1979)，为了有助于该领域研究 的发展, 很有必要对太白红杉种子的萌发特性进行 
研究。目前关于这方面的研究还比较少见。另外, 国内关于落叶松属的种子的研究有 7 种已有较详细 的介绍(国家林业局国有林场和林木种苗工作总站， 2001)。本文不仅是对太白红杉种子的研究, 也是对 我国木本植物种子研究的补充。

\section{1 试验材料和方法}

太白红杉种子采自秦岭太白山南坡海拔 3100 $\sim 3400 \mathrm{~m}$ 处的太白红杉林下, 自然风干。选取健康 饱满的种子进行不同温度条件(恒温和变温)和不同 光照条件(光照和黑暗)的萌发试验。取完好饱满的 种子, 用 $75 \%$ 的酒精浸泡 $10 \mathrm{~min}$ 后, 用蒸馏水冲洗 10 min 然后用 $10 \% \quad \mathrm{NaClO}_{2}$ 浸泡 $20 \mathrm{~min}$ 添加表面活 性剂(吸收液等)少量,用自来水冲洗 $30 \mathrm{~min}$ 。

太白山海拔 $3050 \mathrm{~m}$ 林线处的气候特征表现为 6.7.8 月的最高均温为 $24.5{ }^{\circ} \mathrm{C}$ 最低均温为 $3.7{ }^{\circ} \mathrm{C}$, 日均温为 $11.6{ }^{\circ} \mathrm{C}$ (陈存根等, 1994)。根据太白红杉 分布的这个区域的温度条件设置试验环境条件, 具
体如表 1 。

处理 $1 、 2 、 3 、 4$ 条件下, 当种子充分吸胀后取样 (以露白为标志) , 然后在中国农业大学作物化控室 进行植物激素 ELISA 测试, 测定 4 类激素含量。以 吸胀前的种子作对照。植物激素测定方法为植物激 素 ELISA，在中国农业大学分析测试中心测定。

以滤纸和蒸馏水为萌发基质, 在玻璃培养血(直 径为 $11 \mathrm{~cm}$ )中进行萌发试验。试验在 HPG-400 光照 培养箱中进行。光照强度为 $9000 \mathrm{Lx}$, 湿度为 $70 \%$ ～80\%。每个培养血放 100 粒种子, 每个处理重复 5 次。种子露白开始即为萌发开始, 每 $2 \mathrm{~d}$ 记录萌发 的种子数。以连续 $7 \mathrm{~d}$ 不再有新的种子萌发为试验 结束标志。

发芽率 $=\frac{\text { 种子发芽数 }}{\text { 总种子数 }} \times 100 \%$

发芽指数 $=\sum \frac{G_{t}}{D_{t}}$ (其中, $G_{t}$ 为时间 $t$ 内的发芽数, $D_{t}$ 为相应的发芽日数)

表 1 太白红杉种子萌发试验处理条件

Table 1 Treatments of the experiment

\begin{tabular}{|c|c|c|c|c|c|c|}
\hline $\begin{array}{l}\text { 处理条件 } \\
\text { Treatment }\end{array}$ & $\begin{array}{c}\text { 处理 Treatment } 1 \\
\mathrm{~T} 1\end{array}$ & $\begin{array}{c}\text { 处理 Treatment } 2 \\
\mathrm{~T} 2\end{array}$ & $\begin{array}{c}\text { 处理 Treatment } 3 \\
\text { T3 }\end{array}$ & $\begin{array}{c}\text { 处理 Treatment } 4 \\
\text { T4 }\end{array}$ & $\begin{array}{c}\text { 处理 Treatment } 5 \\
\text { T5 }\end{array}$ & $\begin{array}{c}\text { 处理 Treatment } 6 \\
\text { T6 }\end{array}$ \\
\hline $\begin{array}{l}\text { 温度 } \\
\text { Temperature }\end{array}$ & $\begin{array}{l}\text { 恒温 } 25{ }^{\circ} \mathrm{C} \\
\text { Constant tempera- } \\
\text { ture of } 25{ }^{\circ} \mathrm{C}\end{array}$ & $\begin{array}{l}\text { 恒温 } 25{ }^{\circ} \mathrm{C} \\
\text { Constant tempera- } \\
\text { ture of } 25^{\circ} \mathrm{C}\end{array}$ & $\begin{array}{l}\text { 变温 } \\
\text { Alternanting tem- } \\
\text { perature }\end{array}$ & $\begin{array}{l}\text { 变温 } \\
\text { Alternanting tem- } \\
\text { perature }\end{array}$ & $\begin{array}{l}\text { 恒温 } 12{ }^{\circ} \mathrm{C} \\
\text { Constant tempera- } \\
\text { ture of } 12{ }^{\circ} \mathrm{C}\end{array}$ & $\begin{array}{l}\text { 恒温 } 12{ }^{\circ} \mathrm{C} \\
\text { Constant tempera- } \\
\text { ture of } 12{ }^{\circ} \mathrm{C}\end{array}$ \\
\hline $\begin{array}{l}\text { 光照 } \\
\text { Illumination }\end{array}$ & $\begin{array}{l}24 \mathrm{~h} \text { 光照 } \\
\text { Constant illumi- } \\
\text { nation of } 24 \mathrm{~h}\end{array}$ & $\begin{array}{l}\text { 交替光照 } \\
\text { Alternanting illu- } \\
\text { mination }\end{array}$ & $\begin{array}{l}24 \mathrm{~h} \text { 光照 } \\
\text { Constant illumi- } \\
\text { nation of } 24 \mathrm{~h}\end{array}$ & $\begin{array}{l}\text { 交替光照 } \\
\text { Alternanting illu- } \\
\text { mination }\end{array}$ & $\begin{array}{l}24 \mathrm{~h} \text { 光照 } \\
\text { Constant illumi- } \\
\text { nation of } 24 \mathrm{~h}\end{array}$ & $\begin{array}{l}\text { 交替光照 } \\
\text { Alternanting illu- } \\
\text { mination }\end{array}$ \\
\hline
\end{tabular}

交替光照为 $12 \mathrm{~h}$ 光照 $12 \mathrm{~h}$ 黑暗 Alternanting illumination :photophase is $12 \mathrm{~h}$ and dark phase is $12 \mathrm{~h}$ 变温为 $12{ }^{\circ} \mathrm{C}$ 温度 $12 \mathrm{~h}, 25{ }^{\circ} \mathrm{C}$ 温度 $12 \mathrm{~h}$ Alternanting temperature : temperature of $25{ }^{\circ} \mathrm{C}$ was kept for $12 \mathrm{~h}$ and temperature of $12{ }^{\circ} \mathrm{C}$ for $12 \mathrm{~h}$

\section{2 结果与讨论}

\section{1 种子的萌发率}

在培养血中培养 $12 \mathrm{~d}$ 后种子开始萌发。试验 说明太白红杉种子常温浸种就可以萌发,而松科有 些种的种子的萌发浸种需要高温处理 $\left(50 \sim 60{ }^{\circ} \mathrm{C}\right)$ (于海中等, 1999)。试验结果说明:种子的萌发受 温度的影响较明显, $25{ }^{\circ} \mathrm{C}$ 温度要比 $12{ }^{\circ} \mathrm{C}$ 温度更有 利于种子的萌发。在前 $10 \mathrm{~d}$,处理 1 的种子萌发率 高于处理 2 的; 在 $10 \mathrm{~d}$ 以后，处理 2 的种子萌发率更 高一些(图 1)。在处理 3 和 4 也表现出相同的变化 趋势 (图 2)。这说明在恒温 $25{ }^{\circ} \mathrm{C}$ 和变温 $12{ }^{\circ} \mathrm{C} / 25$ ${ }^{\circ} \mathrm{C}$ 时, 光照与黑暗交替 ( $12 \mathrm{~h}$ 光照 $/ 12 \mathrm{~h}$ 黑暗) 的条件
对于太白红杉种子的萌发是有利的。在较低恒温 $\left(12{ }^{\circ} \mathrm{C}\right)$ 时, 光照与黑暗交替的条件对于太白红杉种 子的萌发的促进作用从试验一开始就表现出来;处 理 6 太白红杉种子的萌发率高于处理 5 的 (图 3)。 在交替光照 ( $12 \mathrm{~h}$ 光照 $/ 12 \mathrm{~h}$ 黑暗) 条件下, 恒温 (25 ${ }^{\circ} \mathrm{C}$ ) 有利于种子萌发 (图 4 ) ; 在 $24 \mathrm{~h}$ 光照条件下, 变 温 (12 h $12{ }^{\circ} \mathrm{C} / 12$ h $25{ }^{\circ} \mathrm{C}$ ) 有利于种子萌发(图 5)。 恒温 $25{ }^{\circ} \mathrm{C}$ 环境中的种子萌发率是高于较低恒温 (12 ${ }^{\circ} \mathrm{C}$ ) 环境和变温环境中的。种子发芽指数 (图 6) 也 表现出相同的趋势。

种子萌发的基本温度必须符合于充分保证植物 幼苗快速发育的外界条件。本研究中的太白红杉是 温带高山植物, 适宜的较高温度 $\left(25^{\circ} \mathrm{C}\right)$ 有利于其种 
子的萌发, 发芽速率随温度的增高而上升, 这与前人 (Larcher, 1975)的研究一致。

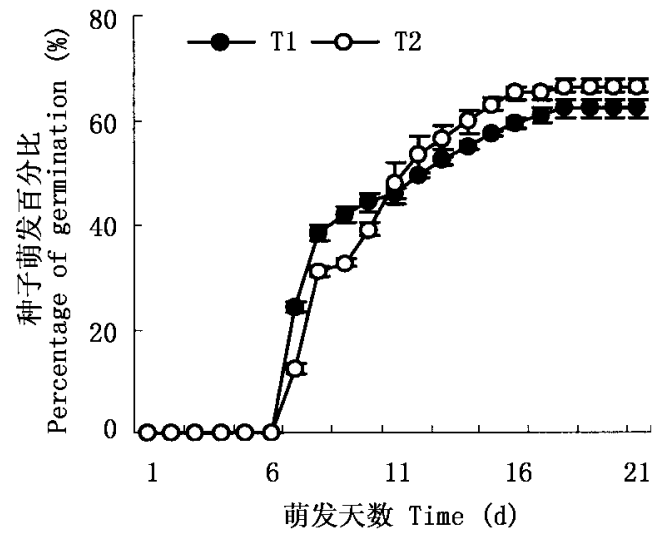

图 1 恒温 $\left(25^{\circ} \mathrm{C}\right)$ 下光照对太白红杉种子萌发的影响

Fig. 1 The effect of light on seed germination of Larix chinensis under condition of constant temperature of $25{ }^{\circ} \mathrm{C}$

$\mathrm{T} 1$,T2 : 同表 1 See Table 1

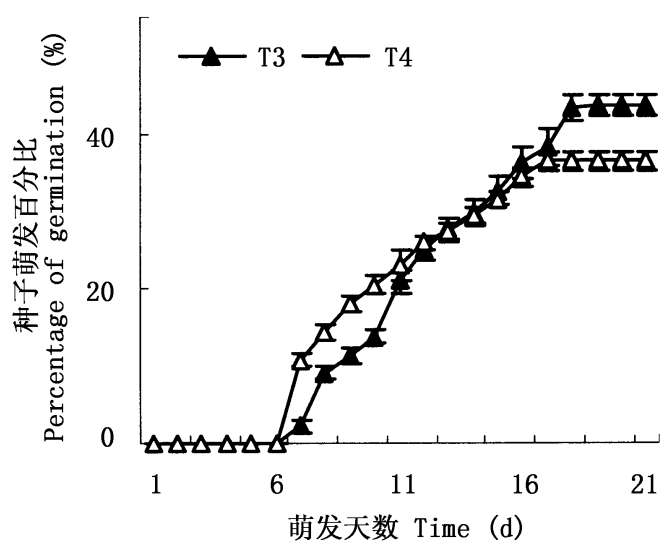

图 2 变温 $\left(12{ }^{\circ} \mathrm{C} / 25^{\circ} \mathrm{C}\right)$ 下光照对太白红杉种子萌发的影响

Fig.2 The effect of light on seed germination of Larix chinensis under condition of alternant temperature of $12{ }^{\circ} \mathrm{C} / 25^{\circ} \mathrm{C}$

$\mathrm{T} 3$,T4 : 同表 1 See Table 1

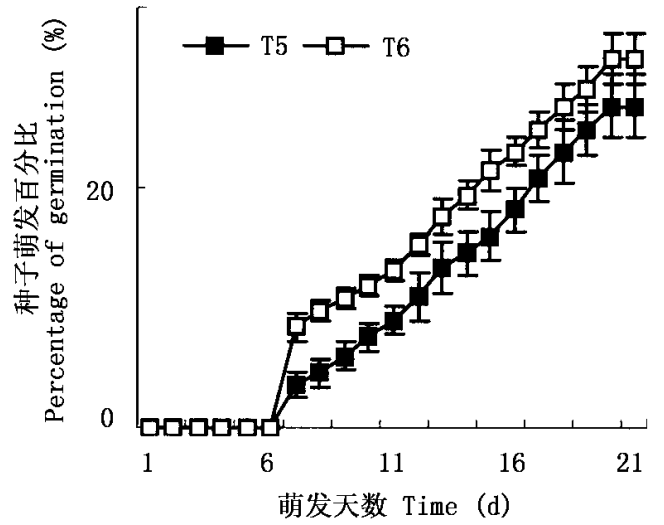

图 3 低恒温 $\left(12{ }^{\circ} \mathrm{C}\right)$ 下光照对太白红杉种子萌发的影响

Fig. 3 The effect of illumination on seed germination of Larix chinensis under condition of constant temperature of $12{ }^{\circ} \mathrm{C}$

T5 ,T6 : 同表 1 See Table 1

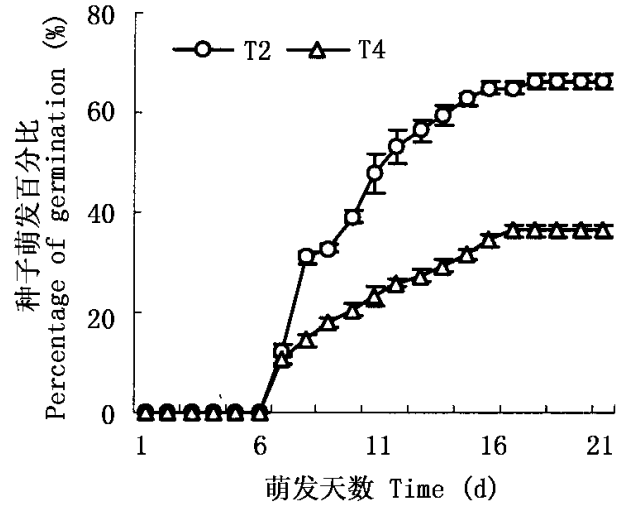

图 4 交替光照 ( $12 \mathrm{~h}$ 光照 $/ 12 \mathrm{~h}$ 黑暗)下温度对 太白红杉种子萌发的影响

Fig.4 The effect of temperature on seed germination of Larix chinensis under condition of alternant illumination (photophase is $12 \mathrm{~h}$ and dark phase is $12 \mathrm{~h}$ )

$\mathrm{T} 2$,T4 : 同表 1 See Table 1

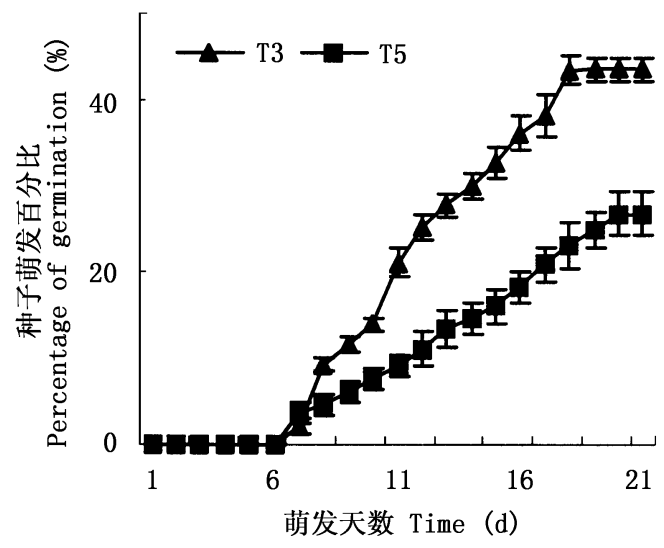

图 $524 \mathrm{~h}$ 光照下温度对太白红杉种子萌发的影响

Fig.5 The effect of temperature on seed germination of Larix chinensis under condition of illumination of $24 \mathrm{~h}$ $\mathrm{T} 3$,T5 : 同表 1 See Table 1

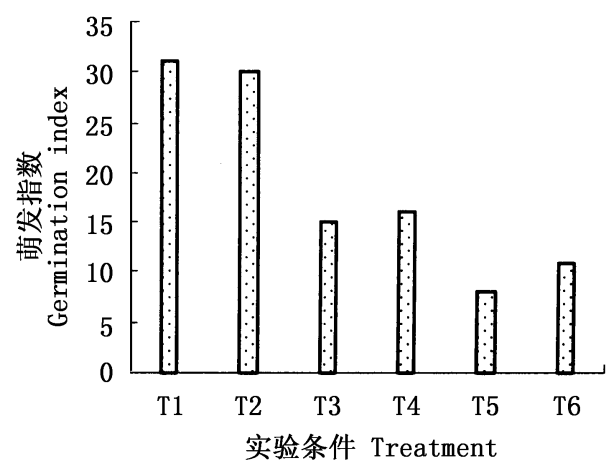

图 6 各试验处理中太白红杉种子发芽指数 Fig.6 Seed germination index of Larix chinensis under every condition

T1 T6 : 同表 1 See Table 1 


\section{2 种子吸胀后的激素含量变化}

种子萌发过程中首先是吸胀阶段, 导致最初形 成的酶释放，随后产生合成蛋白质和 RNA 的能力， 然后开始伸长生长。植物激素与这些变化有联系, 因为在种子萌发过程中, 生长素类有利于细胞壁的 建成和细胞的延伸, 赤霉素类能促进淀粉的分解及
细胞的生长 细胞分裂素类能刺激细胞分裂和分化。 吸胀阶段在种子萌发过程中有着非常重要的作用, 激素的变化可从侧面了解种子在吸胀阶段的生理变 化过程。本试验研究了种子萌发吸胀时的 4 类激素 状况 结果见表 2 。

表 2 种子萌发吸胀时的植物激素含量 (平均值 \pm 标准差)

Table 2 Phytohormone contents in the seed permeated with water of Larix chinensis (Mean $\pm S E$ )

\begin{tabular}{|c|c|c|c|c|c|}
\hline \multirow{2}{*}{$\begin{array}{c}\text { 激素含量 } \\
\text { Phytohormone contents } \\
\left(\mathrm{ng} \mathrm{g}^{-1} \mathrm{FW}\right)\end{array}$} & \multicolumn{5}{|c|}{ 处理 Treatment } \\
\hline & $\begin{array}{c}\text { 对照 Control } \\
\text { CK }\end{array}$ & $\begin{array}{c}\text { 处理 Treatment } 1 \\
\text { T1 }\end{array}$ & $\begin{array}{c}\text { 处理 Treatment } 2 \\
\text { T2 }\end{array}$ & $\begin{array}{c}\text { 处理 Treatment } 3 \\
\text { T3 }\end{array}$ & $\begin{array}{c}\text { 处理 Treatment } 4 \\
\text { T4 }\end{array}$ \\
\hline 生长素 IAA & $910.7 \pm 67.9$ & $148.9 \pm 19.9$ & $138.7 \pm 10.4$ & $89.6 \pm 0$ & $123.1 \pm 2.2$ \\
\hline 赤霉素 GA & $595.3 \pm 22.3$ & $295.6 \pm 63.3$ & $479.8 \pm 19.9$ & $289.8 \pm 48.8$ & $338.4 \pm 25.5$ \\
\hline 脱落酸 ABA & $1487.9 \pm 180$ & $113.2 \pm 2.3$ & $161.3 \pm 3.4$ & $83.4 \pm 6.9$ & $129.6 \pm 16.1$ \\
\hline 细胞分裂素 CTK & $703.8 \pm 126.8$ & $360.1 \pm 96.4$ & $629.3 \pm 55.8$ & $475.2 \pm 39$ & $405.9 \pm 47.7$ \\
\hline
\end{tabular}

$S E:$ Standard error

试验结果表明 种子吸胀后 种子中的植物激素 $(A B A 、 I A A 、 C T K 、 G A)$ ，大部分的含量都明显下降了。 下降幅度最大的是脱落酸 $(\mathrm{ABA})$ 。在对照处理中,$A$ 类激素中 $\mathrm{ABA}$ 的含量最高; 这与 $\mathrm{ABA}$ 的抑制性特 点有关; 因为 $A B A$ 是一种抑制剂，它会抑制种子的 萌发。在成熟种子中, ABA 的作用在于控制萌发维 持休眠 (傅家瑞，1985)。在 4 种处理中, ABA 在处 理 2 中是较高的。处理 1 中 ABA 含量低于处理 2, 处理 4 中 $\mathrm{ABA}$ 含量高于处理 3 。这说明交替光照对 于种子吸胀过程中的 $\mathrm{ABA}$ 有一定的刺激作用。

生长素(IAA)的变化表现为: 处理 $1>$ 处理 $2>$ 处理 $4>$ 处理 3 , 说明在恒温 $\left(25^{\circ} \mathrm{C}\right)$ 条件下, 持续光 照对 IAA 会起一定的刺激作用;而变温条件下 (12 ${ }^{\circ} \mathrm{C} / 25{ }^{\circ} \mathrm{C}$ ) , 交替光照 ( $12 \mathrm{~h}$ 光照 $/ 12 \mathrm{~h}$ 黑暗) 对 IAA 有一定的刺激作用。

细胞分裂素(CTK)的变化表现为:处理 $2>$ 处 理 $3>$ 处理 $4>$ 处理 1 ; 与 IAA 的变化情况相反。 说明在恒温 $\left(25{ }^{\circ} \mathrm{C}\right)$ 条件下, 交替光照 $(12 \mathrm{~h}$ 光照 $/ 12$ $\mathrm{h}$ 黑暗) 刺激 CTK ; 而变温 $\left(12{ }^{\circ} \mathrm{C} / 25{ }^{\circ} \mathrm{C}\right)$ 条件下，持 续光照对 CTK 有刺激作用。据报道: 未成熟种子 中 细胞分裂素含量较高, 而干燥种子难以测出; 也 有研究表明: 莴苣种子在发芽初期不含 CTK, 发芽 $24 \mathrm{~h}$ 后便会产生(曹宗巽, 1980)。在本试验中, 无论 是干种子, 还是发芽中的种子, 都有细胞分裂素测 出。细胞分裂素含量的增加有助于细胞旺盛分裂和 分化, 与种子的萌发生理有一定的一致性。Wheeler (1960) 早就认为在种子萌发的早期可能存在赤霉 素。本试验中干种子中含有较多的赤霉素。GA 的
变化表现为 :处理 $2>$ 处理 $4>$ 处理 $1>$ 处理 3 。 说明在恒温 $\left(25{ }^{\circ} \mathrm{C}\right)$ 条件和变温条件 $\left(12{ }^{\circ} \mathrm{C} / 25{ }^{\circ} \mathrm{C}\right)$ 两种情况中, 交替光照 ( $12 \mathrm{~h}$ 光照 $/ 12 \mathrm{~h}$ 黑暗) 对 $\mathrm{GA}$ 有刺激作用。

在恒温和变温两种情况中, 交替光照对于种子 吸胀后的 ABA、GA 有一定的刺激作用。在恒温条 件下，持续光照对于种子吸胀后的 IAA 有一定的刺 激作用, 而变温条件下交替光照对生长素有刺激作 用。而 CTK 的变化情况正好与 IAA 相反。

试验结果还表明 :光照条件相同时，恒温条件下 的植物激素含量要高于变温条件下的, 说明恒温对 各种激素有刺激作用。对照发芽试验, 发现恒温(25 $\left.{ }^{\circ} \mathrm{C}\right)$ 条件下的萌发率高于变温 $\left(12{ }^{\circ} \mathrm{C} / 25{ }^{\circ} \mathrm{C}\right)$ 条件下 的种子萌发率, 这也反映了内源激素在种子萌发过 程中起着重要作用。

\section{3 结 语}

由于林线与环境的关系非常复杂，林线的形成 原因也可以说多种多样。在关于高山林线形成原因 的诸多科学研究解释中, 有一种重要的观点是热量 或温度方面解释。有人曾认为温度对高山林线有着 决定性影响 (Tranquillini， 1979 ; Daubenmire，1954)。 Shaw (1909) 曾认为积雪的存在延误了林线内草甸下 层树木种子的顺利生长。但是温度对植物生长的影 响也相当复杂, 并非通常所认为的线性形式影响 (Tranquillini , 1979)。

本研究试验结果显示: 温度对于太白红杉种子 的萌发率和种子吸胀时的激素有重要作用; ;内源激 
素在太白红杉种子萌发过程中起着重要作用,也会 影响到种子萌发后植物的生长。相比之下光照的作 用就很小。因此在野外, 温度可能是影响太白红杉 种子萌发的一个至关重要的因子。由于温度与光照 条件影响太白红杉种子在土壤中的萌发,从而影响 植被的更新。环境因子是综合作用于林线植被的， 同时具有一定的区域性 ;目前本文的研究是初步的， 温度和光照与太白山林线的成因之间的关系仍有待 于进一步研究。

\section{参 考 文 献}

Cao,Z. X. (曹宗巽) . 1980. Plant physiology. Beijing: People’s Education Press. 190 193. (in Chinese)

Chen, C. G. (陈存根) \& H. Peng (彭鸿). 1994. The phytocoenological features and classification of the forests of Larix chinensis in Qinling range. Scientia Silvae Sinicae(林业科学), 30: 487 496. (in Chinese with English abstract)

Daubenmire, R. 1954. Alpine timberline in the Americas and their interpretation. Bulter University Botanical Studies, 11: 119 136

Fu, J. R. (傅家瑞). 1985. Seed physiology. Beijing: Science Press. $333 \sim 334$. (in Chinese)

Larcher, W. 1975. Physiological plant ecology. Berlin, Heidel- berg, New York: Springer-Verlag. $237 \sim 272$.

National Service Center of State-Owed Forest Farms and Forest Seed and Seedling Affairs of the Forestry Ministry. (国家林业局国有 林场和林木种苗工作总站). 2001. Seeds of woody plants in China. Beijing: China Forestry Publishing House. 29 34. (in Chinese)

Shaw, C. H. 1909. The cause of timberline on mountains. Plant World, 12:169 181.

Thompson, K. 1992. The functional ecology of seed banks. In: Fenner, M. ed. Seeds the ecology of regeneration in plant communities. Wallingford: CAB International. $231 \sim 258$.

Tranquillini, W. 1979. Physiological ecology of alpine timberline. Berlin, New York: Springer-Verlag.

Wheeler, A. W. 1960. Changes in leaf growth substance in cotyledons and primary leaves bean seedlings. Journal of Experimental Botany, 11: $217 \sim 226$.

Yu, H. Z. (于海中), Y. L. Tian(田艳丽), Y. J. Niu(牛永 杰) \& X. Feng (冯旭). 1999. Study on the morphology of Larix gemelini, Larix olgensis and Larix principis-rupprechtii. Forestry Science and Technology (林业科技), 24 (6): 1 4. (in Chinese with English abstract)

Zhang, L. (张玲) \& J. Y. Fang (方精云). 2004. Composition and dominance species of soil seed bank along an alpine altitudinal gradient in Mt. Taibai, Qinling Mountains. Biodiversity Science (生物多样性), 12: 123 130. (in Chinese with English abstract) 